\title{
Bacterial magnetosomes as an efficient gene delivery platform for cancer theranostics
}

\author{
Qinglei Dai ${ }^{1}$, Ruimin Long ${ }^{1,3}$, Shibin Wang ${ }^{1,2,3}$, Ranjith Kumar Kankala1,3, Jiaojiao Wang ${ }^{4}$, Wei Jiang ${ }^{4}$ \\ and Yuangang Liu Li,2, $^{*}$
}

\begin{abstract}
Background: Gene therapy has gained an increasing interest in its anti-tumor efficiency. However, numerous efforts are required to promote them to clinics. In this study, a novel and efficient delivery platform based on bacterial magnetosomes (BMs) were developed, and the efficiency of BMs in delivering small interfering ribonucleic acid (siRNA) as well as antiproliferative effects in vitro were investigated.

Results: Initially, we optimized the nitrogen/phosphate ratio and the BMs/siRNA mass ratio as 20 and 1:2, respectively, to prepare the BMs-PEI-siRNA composites. Furthermore, the prepared nanoconjugates were systematically characterized. The dynamic light scattering measurements indicated that the particle size and the zeta potential of BMs-PEl-siRNA are $196.5 \mathrm{~nm}$ and $49.5 \pm 3.77 \mathrm{mV}$, respectively, which are optimum for cell internalization. Moreover, the confocal laser scanning microscope observations showed that these composites were at a proximity to the nucleus and led to an effective silencing effect. BMs-PEI-siRNA composites efficiently inhibited the growth of HeLa cells in a dose-as well as time-dependent manner. Eventually, a dual stain assay using acridine orange/ethidium bromide, revealed that these nanocomposites induced late apoptosis in cancer cells.
\end{abstract}

Conclusions: A novel and efficient gene delivery system based on BMs was successfully produced for cancer therapy, and these innovative carriers will potentially find widespread applications in the pharmaceutical field.

Keywords: Bacterial magnetosomes, siRNA, Polyethylenimine, Gene therapy

\section{Background}

Cancer is one of the leading causes of deaths, accounting for millions of deaths annually. More often, chemotherapy is the primarily advised therapeutic regimen after surgery and/or radiation therapy to improve the survival rate in patients with cancer. However, most of the chemotherapeutic agents result in several adverse effects due to their non-specific uptake by healthy cells, poor bioavailability and multidrug resistance (MDR) attained by cancer cells, among others [1]. In addition, these undesired effects result in the lower therapeutic efficacy of conventional chemotherapeutic agents. To this end, gene therapy has shown a great potential in the treatment of many cancers because of the ability of genes in eradicating the

\footnotetext{
${ }^{*}$ Correspondence: ygliu@hqu.edu.cn

${ }^{3}$ Fujian Provincial Key Laboratory of Biochemical Technology, Huaqiao

University, Xiamen 361021, People's Republic of China

Full list of author information is available at the end of the article
}

hereditary diseases and replace the defective cell specifically [2, 3]. Moreover, the small (or short)-interfering ribonucleic acid (siRNA), often called as silencing RNA, is a class of chemically synthesized double-stranded RNA molecules with 19-23 nucleotides that can trigger the silencing of homologous gene expression [4]. Inspired by this fact, the researchers have harnessed the siRNA for various applications in biomedical field [5, 6]. However, there has not been an anticipated success for their exploration in clinics, due to various reasons such as lack of stability in organism caused by ribonuclease (RNase) degradation, poor cellular uptake, and endosomal trapping, among others [7]. To overcome these issues, a wide-variety of non-viral vectors have been used to deliver siRNA, including lipid, cationic polymers, and inorganic nanoparticles, which are advantageous over virus-based vectors.

Out of various non-viral vectors available, cationic polymers have gained the significant importance for the 
efficient conveyance of genes due to their advantages such as high stability in physiological fluids, controlled release of active pharmaceutical agents (APIs) including genes, large capacity of gene packing and ease of structural modification to improve the transfection efficiency and stability of genes. Polyethyleneimine (PEI) is one of the most promising polymeric substrates explored for the efficient delivery of DNA [8, 9], siRNA [10, 11], and oligonucleotides [12]. More often, the genes with desired nucleotide sequences are encapsulated in the PEI through electrostatic interactions. In addition, the interesting feature of PEI is that it condenses the anionic siRNA and subsequently protects the siRNA from degradation by RNase [13]. Preceding research has indicated that PEI can prevent exocytosis through the proton sponge effect, which induces the flow of chloride ions and thereby promotes the osmotic swelling of endosomes/lysosomes and subsequently releases the APIs [14]. Despite its efficiency in delivery, several factors of PEI significantly affect the transfection efficiency and toxicity of genes such as the molecular weight and structure of PEI. In addition, advancements in the PEI-based design are still obligatory to achieve the efficient delivery of genes by reducing the adverse effects simultaneously.

Pharmaceutical carriers often use polymers, dendrimers, micelles, liposomes, inorganic nanomaterials and so on [15-21], which can all be employed for drug delivery system. Amongst inorganic nanomaterials, bacterial magnetosomes (BMs) have shown a great potential as a novel carrier due to their excellent biocompatibility, high surface area to volume ratio, superparamagnetism and abundant active sites on the membrane of BMs [22]. More often, BMs are extracted from magnetotactic bacteria with magnetic iron oxide or iron sulfide enclosed by a natural phospholipid membrane [23], which endowed them with high biocompatibility. In a case, the purified and sterilized BMs have shown that they were non-toxic to mouse fibroblasts in vitro [22]. In addition, the pyrogen test revealed that the administered BMs (1 mg) resulted in no significant change in the body temperature of rabbits. In another study, Sun et al. evaluated the acute toxicity, immunotoxicity, and cytotoxicity of BMs [24]. The blood examination results of BMs have shown no significant effect compared to the control group of rats. However, BMs showed a slight cytotoxicity in H22, HL60, or EMT6 cell lines. In recent times, BMs have gained an increasing interest for the delivery of proteins, chemotherapy drugs and DNA [25-27].

Motivated by these facts, this study reports the synthesis of gene delivery system based on BMs for the effective delivery of siRNA by using PEI as a crosslinker (BMsPEI-siRNA). Furthermore, various techniques were used to systematically characterize the nanocomposites such as transmission electron microscope (TEM) for morphology, DLS measurements for particle size distribution and others. Furthermore, the stability and bioactivity studies were performed to elucidate the integrity and the anti-proliferative effects of the siRNA-loaded BMs, respectively.

\section{Results and discussion}

\section{Characterization of BMs and its conjugates}

From the TEM images (Fig. 1), it is evident that the particle sizes and morphology of BMs are uniform and the diameter was between 30 and $50 \mathrm{~nm}$ with the hexagonal arrangement (Fig. 1a). It reflects the coated layer over the BMs indicating that the distinct membrane was
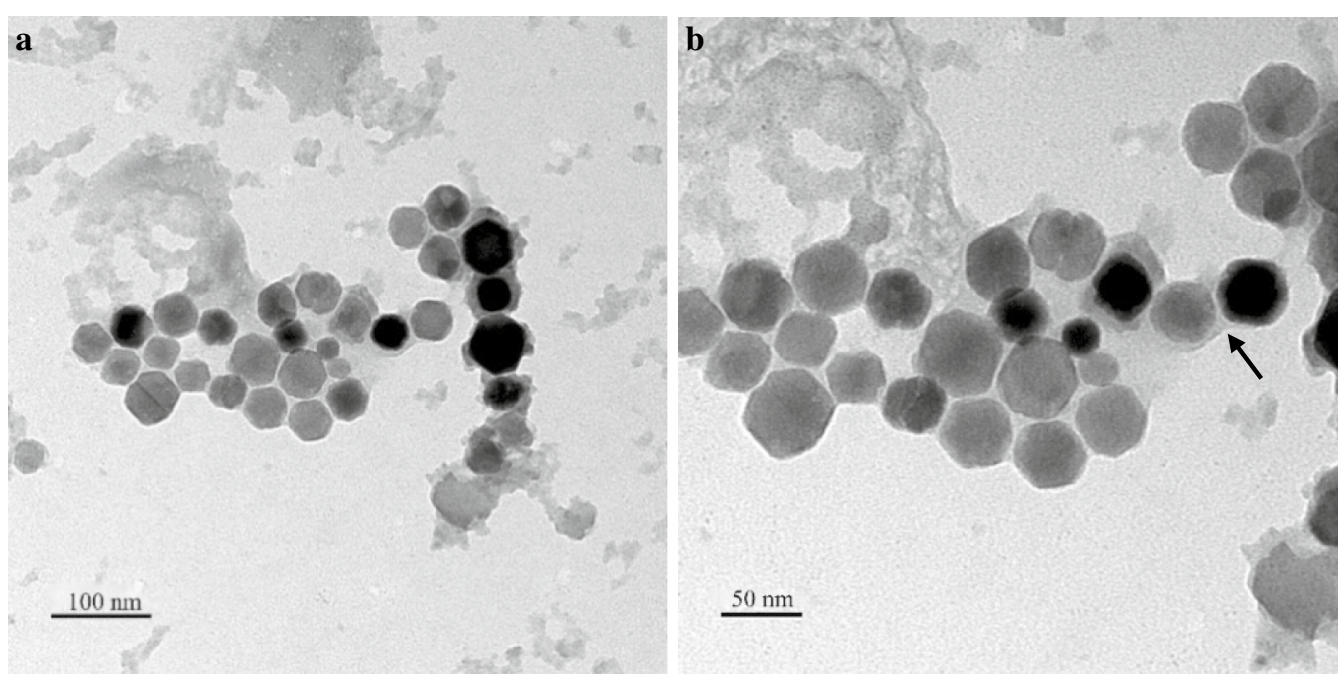

Fig. 1 TEM images of BMs (black arrows indicating the uniform and clear lipid membrane of BMs) 
composed of phospholipids and fatty acids [28]. Furthermore, the zeta potential value of BMs was measured by adjusting the $\mathrm{pH}$ of the sample to physiological $\mathrm{pH}$ (7.4). The surface charge of BMs was $-48.3 \pm 2.6 \mathrm{mV}$, owing to the existence of abundant lipids and amino groups [29]. However, the negative surface charge is countered with a positively-charged PEI for the efficient loading of genes, which condenses the anionic siRNA molecules, and interacts with negatively-charged BMs via electrostatic interactions.

To optimize the formulation of BMs-PEI-siRNA, we performed agarose gel electrophoresis of samples with different N/P ratios concerning the nitrogen in PEI and phosphates in siRNA respectively, and the results were shown in Fig. 2a. The N/P ratio at which the undetected fraction of free siRNA demonstrates that the siRNA was successfully bound to PEI via electrostatic interactions. The retardation efficiency was increased with the increase in N/P ratio. Eventually, the optimized N/P ratio was found above 8 , and the samples of BMs-PEI-siRNA composites were prepared at that ratio and systematically characterized using various techniques.

The dynamic light scattering (DLS) measurements gave the hydrodynamic mean diameters as well as zeta potential values of BMs-PEI-siRNA composites. As shown in Fig. 2c, e, the diameters of the synthesized nanocomposites decreased with the increase of N/P ratios, demonstrating that the addition of siRNA and PEI complex resulted in the compact nanocomposites. Moreover, the zeta potential values of respective nanocomposites were in a positive range, which confirms the conjugation of PEI. The hydrodynamic diameter and the positive potential of BMs-PEI-siRNA formulation with N/P ratio 20 $(200 \mathrm{~nm})$ are optimum for the accumulation through enhanced permeation and retention (EPR) effect and ease of cellular internalization in the tumor [30].

Further, we investigated the concentration of BMs that would be effective in the formation of BMs-PEI-siRNA through agarose gel electrophoresis. The concentration of BMs at which the gel devoid of free siRNA band indicates that they have no significant effect on the immobilization. Figure $2 \mathrm{~b}$ shows the gel images of BMs-PEI-siRNA composites with different BMs/siRNA weight ratios, at which the bandwidth of BMs/siRNA weight ratio 1:2 was found optimum. Moreover, the size was relatively small (Fig. 2f) and the potential was positive (Fig. 2d), at this ratio, which was extremely beneficial for establishing the interactions with the cell and subsequent internalization process.

\section{Stability studies}

siRNA is one of the most sensitive biomolecules in the body, which suffer from certain limitations during delivery such as short circulation times, reduced therapeutic effects and others due to in vivo degradation [31]. In addition, a few factors such as serum proteins are considered during the formulation of genes for cancer theranostics. Herewith, we demonstrated the stability of our design through various methods such as decomplexation assay, enzyme stability assay, and others. In heparin decomplexation assay, the siRNA dissociates from the synthesized nanocomposites due to the stronger interaction of the heparin with the nanocomposites. The experiment was performed by mixing various concentrations of heparin with the nanocomposites, and the resultant siRNA in the supernatant was subjected to gel electrophoresis. The results (Fig. 3a) indicated that the siRNA was utterly dissociated from the nanocomposites at a specific weight ratio of heparin to siRNA (10:1) after incubating for $15 \mathrm{~min}$. Figure $3 \mathrm{~b}$ elucidates the stability of siRNA in the presence of serum proteins. It is evident that the naked siRNA was degraded rapidly in 50\% FBS for $60 \mathrm{~min}$, while the fraction can be still observed in the case of BMs-PEI-siRNA nanocomposites after $150 \mathrm{~min}$ of exposure, demonstrating that the designed nanocomposites offered significant protection to siRNA. Eventually, the stability of siRNA in the presence of enzymes was demonstrated by suspending the designed nanocomposites in the presence of RNase A. The results showed that the immobilization of siRNA in the PEI network on BMs significantly reduced the degradation, which can enhance the circulation time in vivo.

\section{Cell viability assay}

The anti-tumor efficacy of our novel BMs-based gene delivery system was performed using CCK- 8 assay in the HeLa cell line. The experiments were designed such that they represent both dose-dependent by treating various doses and time-dependent cytotoxicity by measuring the viability at different time points. In the dosedependent assay, the viability of cells gradually decreased with the increase in the concentration of nanocomposites (Fig. 4a). Moreover, the inhibition rate of BMs-PEIsiRNA (STAT 3) composites at a dose of 10 pmol was significantly higher than that of siRNA alone accounting for $70 \%$ of cell death. The inhibition effect of BMsPEI-siRNA (NC) was similar to that of BMs-PEI vector, indicating that the cytotoxicity of siRNA was sequencespecific. Based on these results, the nanoconjugates at a concentration of 5 pmol were chosen as an optimized dose for further investigations.

As shown in Fig. 4b, BMs-PEI, and BMs-PEI-siRNA (NC) showed no apparent growth inhibition of cells within the incubation times, indicating that the BMs-PEI composites resulted in low toxicity. Cytotoxicity of BMsPEI-siRNA (STAT 3) sample showed the time-dependent 
$\mathbf{a}$

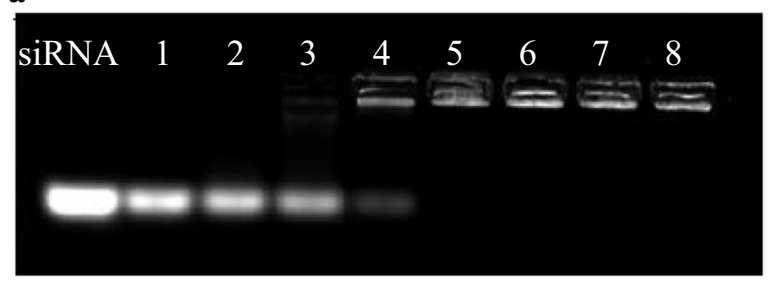

c

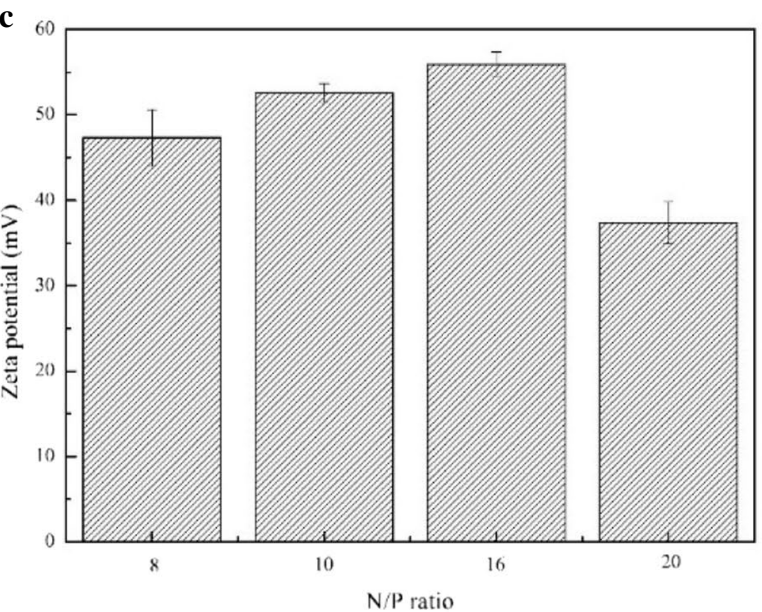

b

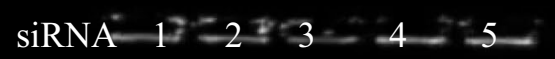

d
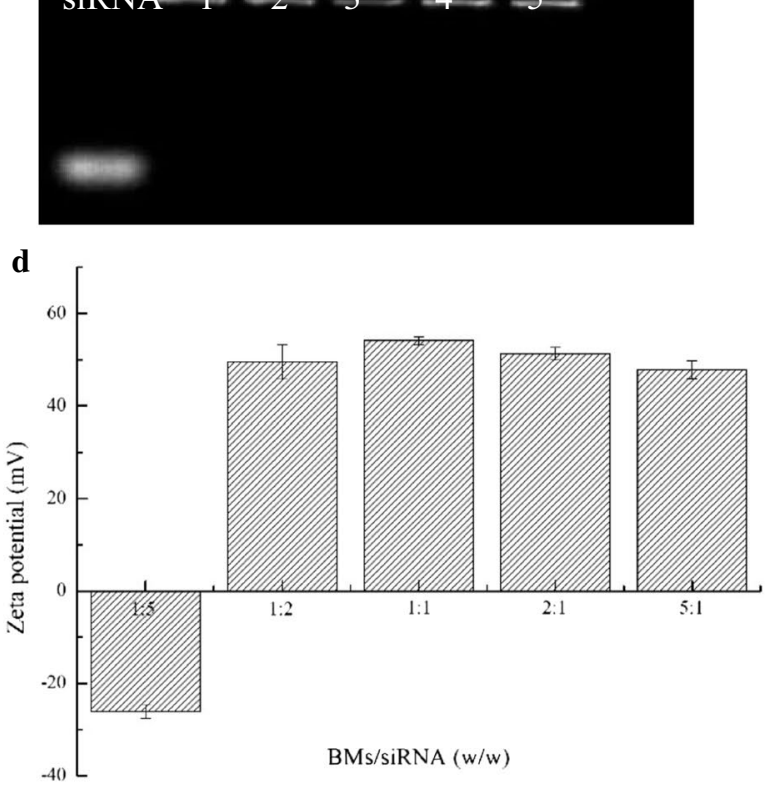

e

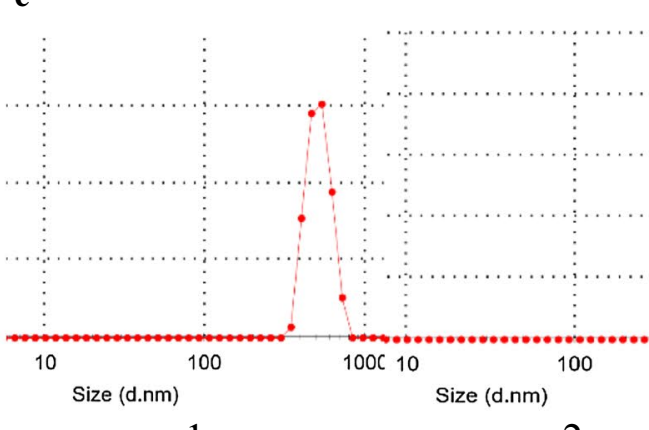

1

2

$562.8 \mathrm{~nm}$

$679.5 \mathrm{~nm}$

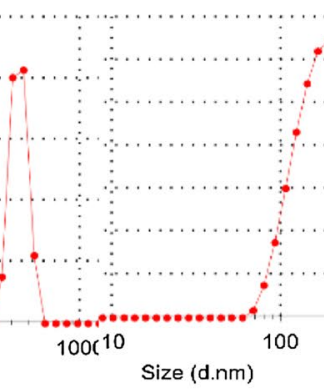

3

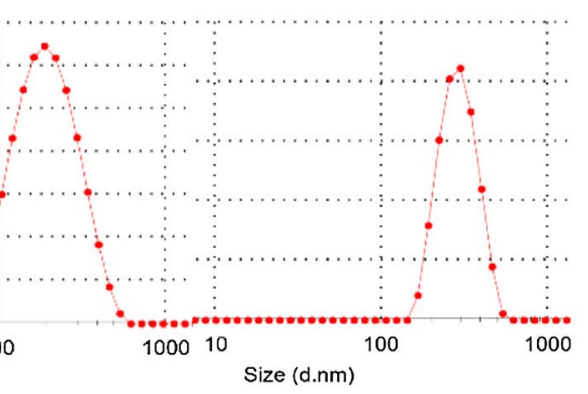

$313.5 \mathrm{~nm}$

4

$277.9 \mathrm{~nm}$

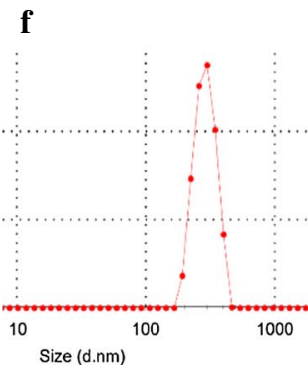

1

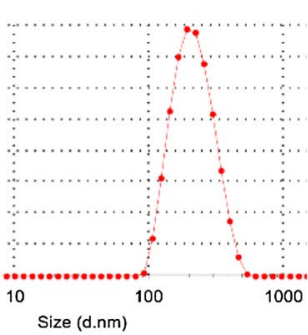

2

$175.6 \mathrm{~nm}$

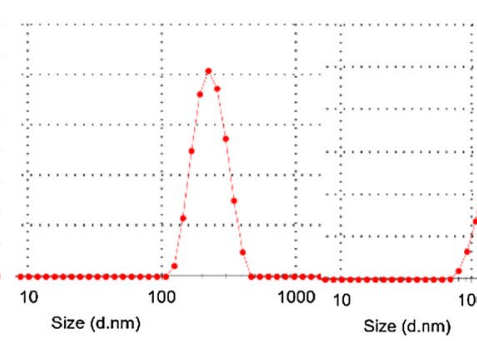

3

$196.5 \mathrm{~nm}$

$234.8 \mathrm{~nm}$

4

$242.9 \mathrm{~nm}$

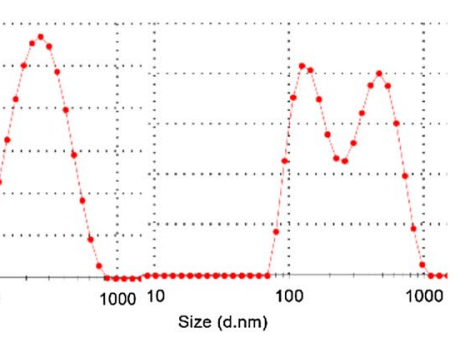

5

Fig. 2 Physical characterization of BMs-PEI-siRNA nanocomposites including the agarose gel electrophoresis results for optimization of formulation. Images showing the gel electrophoresis results of BMs-PEI-siRNA composites $\mathbf{a}$ at different N/P ratios, 1-8: 0, 1, 2, 4, 8, 10, 16, 20; and $\mathbf{b}$ at different BMs/siRNA weight ratios, 1-5: 1:5, 1:2, 1:1, 2:1, 5:1 along with free siRNA; zeta potential values of BMs-PEI-siRNA composites c at different N/P ratios; and $\mathbf{d}$ at different BMs/siRNA mass ratios; hydrodynamic diameters of BMs-PEI-siRNA composites e at different N/P ratios, 1-4: 8, 10, 16, 20; f at different BMs/siRNA ratios, 1-5: 1:5, 1:2, 1:1, 2:1, 5:1 


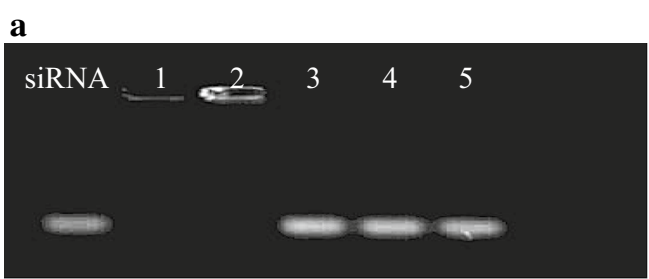

b

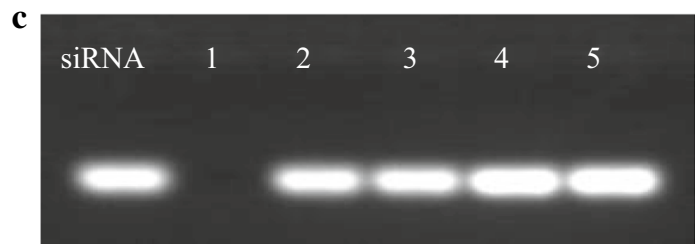

Fig. 3 Agarose gel electrophoresis assay of BMs-PEI-siRNA composites. a The heparin decomplexation assay. 1: Naked siRNA; 2-5: BMs-PEI-siRNA at different heparin/siRNA weight ratio: 2, 10, 25, 100, respectively; b the serum stability, 1: naked siRNA incubation with 50\% FBS for 60 min; 2-5: BMs-PEI-siRNA incubation with 50\% FBS for 60, 90, 120, 150 min, respectively; c the enzyme stability of BMs-PEI-siRNA: 1: naked siRNA incubation with RNase A for 60 min; 2-5: BMs-PEI-siRNA incubation with RNase A for 60, 90, 120, 150 min, respectively

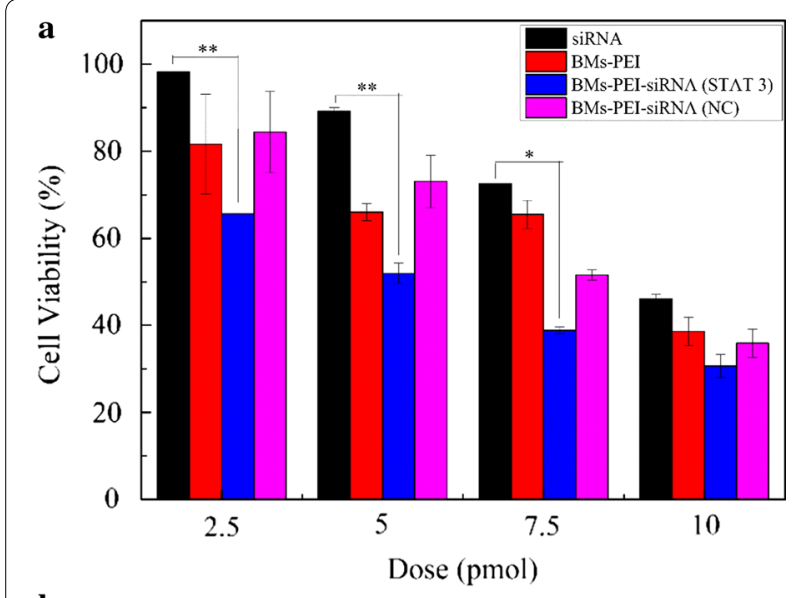

b

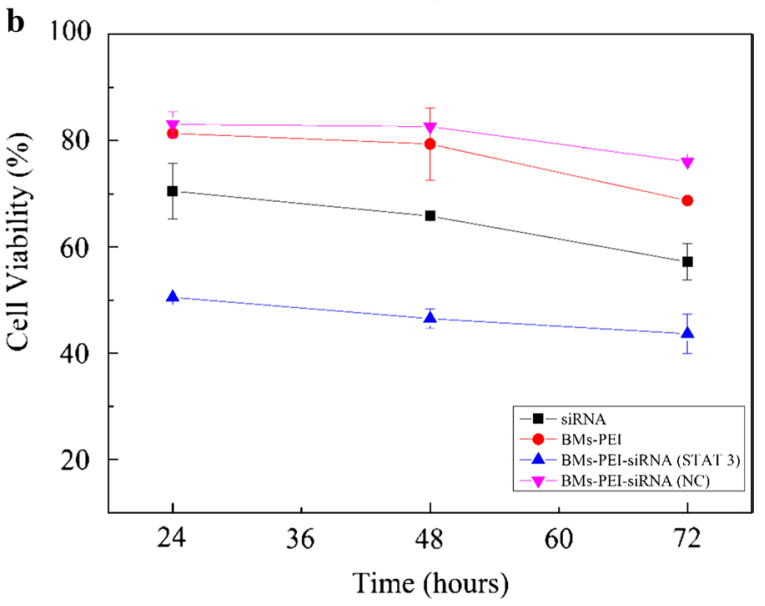

Fig. 4 Cytotoxic effects of BMs-PEI-siRNA nanocomposites. a Dosedependent inhibition and $\mathbf{b}$ time-dependent inhibition of siRNA, BMs-PEI, BMs-PEI-siRNA (STAT 3) and BMs-PEI-siRNA (NC) on HeLa cells $\left.{ }^{*} p<0.05 ;{ }^{* *} p<0.01\right)$ inhibitory effect on HeLa cells about $40 \%$ after 72 h of incubation and was significantly higher compared to that of siRNA treatment group of cells.

\section{Cell apoptosis assay}

To further assess the anti-tumor effect of BMs-PEIsiRNA composites, the cell apoptosis of designed sample was examined using acridine orange/ethidium bromide (AO/EB) dual stain. As shown in Fig. 5, cells in the negative control group were green in color elucidating no apparent cell apoptosis. Comparatively, the cells in siRNA, as well as BMs-PEI-siRNA treated groups indicated more orange stained cells demonstrating that the cells underwent early and late apoptosis. The results were consistent with that of anti-tumor efficacy. Overall, siRNA-loaded BMs-PEI delivery system not only efficiently expressed the silencing effect of siRNA but also induced the apoptosis compared to the naked siRNA.

\section{Cellular uptake of BMs-PEI-siRNA nanocomposites}

Indeed, cellular uptake of nanoparticles plays a crucial role during the formulation of nano-delivery systems for the efficient delivery of therapeutic cargo. However, the positively-charged BMs-PEI nanoparticles are highly suitable for delivering siRNA as they interact with the negatively-charged cell membrane. To validate the internalization of designed nanocarriers, we labeled FAM to siRNA and tracked the presence of nanocomposites using confocal laser scanning microscope (CLSM) in HeLa cells after incubation for 0.5 and 6 h (Fig. 6). Interestingly, the BMs-PEI-siRNA nanocomposites were at the proximity of the nucleus in cells. In addition, some characteristic changes associated with the apoptosis 

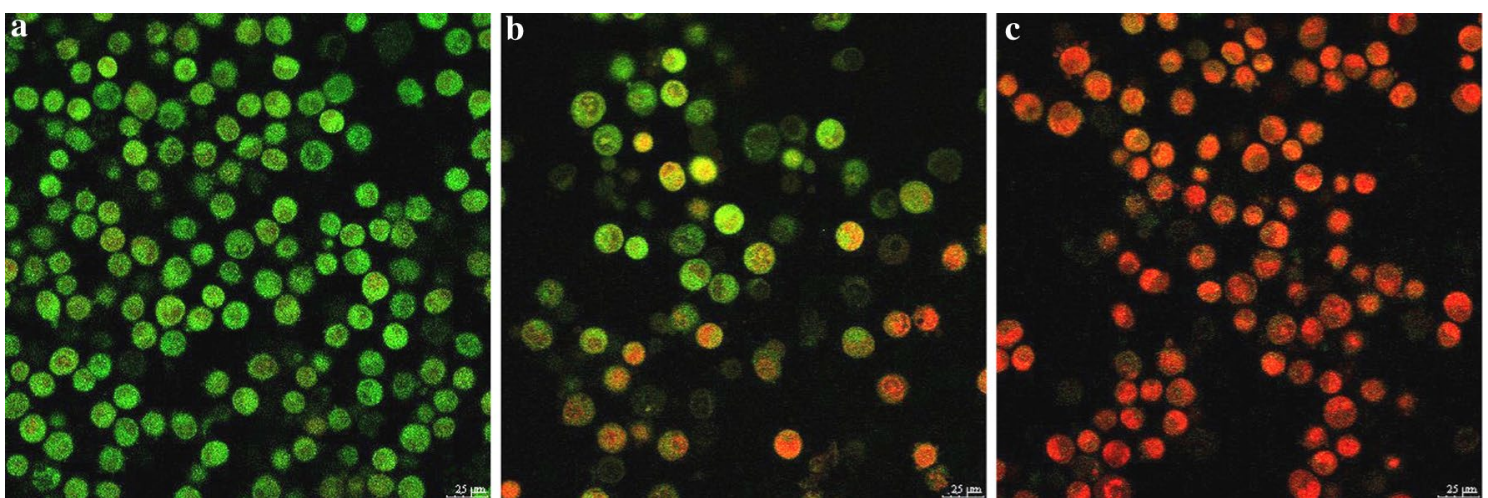

Fig. $5 \mathrm{AO} / \mathrm{EB}$ dual staining of HeLa cells after culturing for $48 \mathrm{~h}$. a Control (media alone); b naked siRNA; c BMs-PEl-siRNA
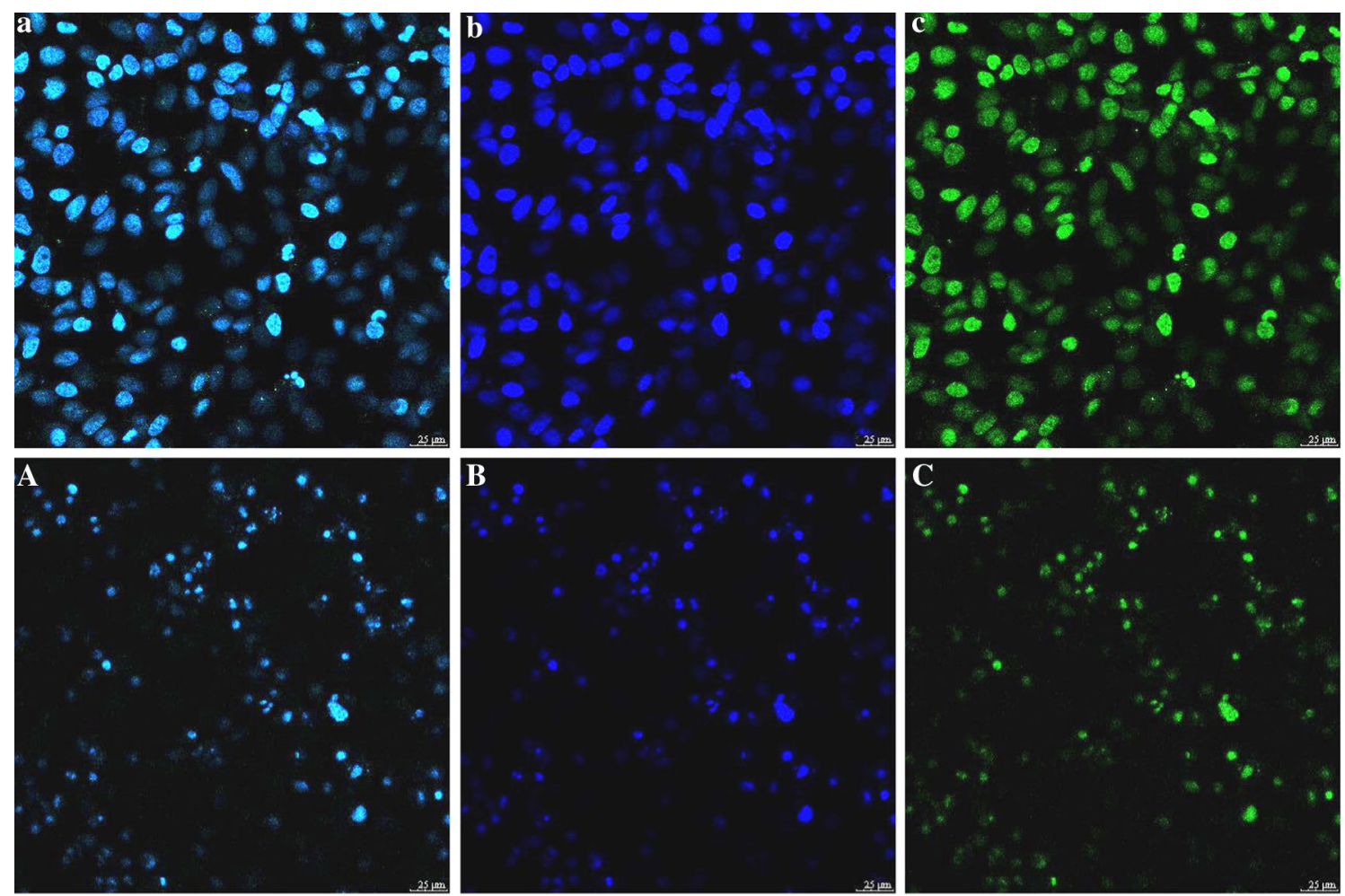

Fig. 6 CLSM images illustrating the cellular uptake of our designed nanocomposites (a-c $0.5 \mathrm{~h}, \mathbf{A}-\mathbf{C} 6 \mathrm{~h})$

such as chromatin condensation and nucleus shrinkage were observed in the cells treated with BMs-PEI-siRNA nanocomposites, indicating that the cells underwent apoptosis. This phenomenon was different from the results of cell uptake in most previous studies [32, 33]. Preceding reports indicated that the RNA interference (RNAi) occurs in the cytoplasm, while other studies have revealed that potent RNAi expressed in the nucleus of human cells [34]. The reason for these contrast findings might be the altered dynamics and distribution of siRNA due to the presence of BMs, which promoted their delivery close to the nucleus.

\section{Conclusions}

In summary, we designed a novel delivery system based on siRNA-loaded BMs using cationic PEI as a crosslinker. After improving the synthetic conditions, the optimal BMs-PEI-siRNA nanocomposites have shown an enhanced cellular uptake and exhibited serum stability as well as enzymatic hydrolysis. These stable 
nanocomposites resulted in more significant inhibitory effects on HeLa cells. This delivery system takes advantage of efficient delivery of siRNA into cancer cells, and also provides an opportunity for the development of various novel therapeutic strategies.

\section{Experimental section}

\section{Materials}

BMs extracted from Magnetospirillum gryphiswaldense MSR-1 were presented kindly by professor Li Ying and Jiang Wei (Department of Microbiology, China Agricultural University). STAT 3 siRNA and siRNA (NC) were purchased from GenePharma Co., Ltd. (Shanghai, China), siRNA (NC) was used as the negative control of STAT 3 siRNA without homology. Branched PEI (MW: $25 \mathrm{KD}$ ) was purchased from Sigma Aldrich (USA). The cervical carcinoma cell line (HeLa cells), was obtained from China Academy Typical Culture Preservation Committee Cell Library (Shanghai, China). Cell culture medium was composed of Dulbecco's Modified Eagle's Medium (DMEM) supplemented with 10\% fetal bovine serum (FBS). The cells were incubated in humidified air maintained at $37^{\circ} \mathrm{C}$ with $5 \% \mathrm{CO}_{2}$.

\section{Preparation and characterization of BMs}

The extraction process of BMs was performed by following our reported procedure given below [35]. Microbial cells of M. gryphiswaldense MSR-1 were suspended in phosphate buffered saline (PBS, $0.1 \mathrm{M}, \mathrm{pH} 7.4$ ) and then the cell membrane was disrupted by ultrasonication. The cell debris was removed by magnetic adsorption, and the process was repeated for about 20 times. The resultant suspension of BMs was treated with DNase I for $2 \mathrm{~h}$ at $37^{\circ} \mathrm{C}$. The BMs were then washed for about 20 times and conserved at $-20{ }^{\circ} \mathrm{C}$ after being freeze-dried. Further, the suspension of BMs was subjected to physical characterization.

The morphology of BMs was confirmed by capturing images using TEM. The zeta potential and particle size distribution of $\mathrm{BMs}$ were measured by Zetasizer (ZEN3600, Malvern Instruments Ltd, UK).

\section{Preparation of BMs-PEI-siRNA nanocomposites}

The nanocomposites with different nitrogen of $\mathrm{PEI} / \mathrm{phos}$ phate of siRNA (N/P) ratios (N/P ratios were set as 0 , $1,2,4,8,10,16$ and 20) were prepared by mixing a certain amount of siRNA with PEI in diethyl pyrocarbonate (DEPC) water, and fixed amounts of BMs were added (BMs/siRNA mass ratio was 1:2), followed by vortexing for $2 \mathrm{~min}$ and incubated for $25 \mathrm{~min}$ at room temperature. The synthetic process of the composites was demonstrated in Fig. 7.

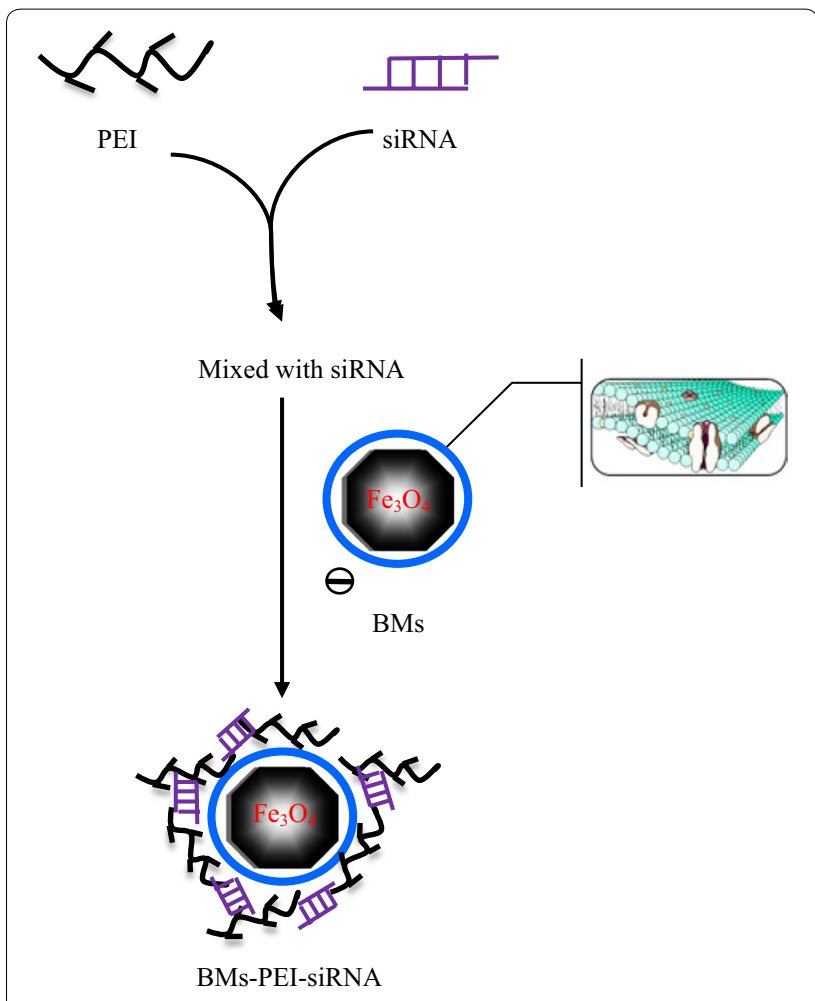

Fig. 7 Schematic illustration showing the synthetic outline of BMsPEI-SiRNA

To obtain the optimal weight ratio of BMs to siRNA, various amounts of BMs were added to the PEI/siRNA $(\mathrm{N} / \mathrm{P}=20)$ complexes in DEPC water (weight ratios of BMs to siRNA were set as 1:5, 1:2, 1:1, 2:1 and 5:1), and incubated for 25 min to obtain BMs-PEI-siRNA nanocomposites. The binding ability was estimated by agarose gel retardation assay.

\section{Electrophoresis assay}

The agarose gel electrophoresis assay was performed to estimate the encapsulation efficiency of siRNA in BMsPEI-siRNA nanocomposites. The resultant nanocomposites were loaded on $0.8 \%(\mathrm{w} / \mathrm{v})$ agarose gel containing $1 \%(\mathrm{v} / \mathrm{v})$ Gel Stain in tris acetate EDTA (TAE) buffer, and the gel was run at $70 \mathrm{~V}$ for $20 \mathrm{~min}$. The gel image was captured using UV transilluminator and a digital imaging system (GIS2008, Tanon Science \& Technology Co., Ltd, China).

\section{Stability studies}

The stability of designed nanocomposites was determined by incubating them in various conditions provided, which mimic the physiological fluids. One of them was the heparin decomplexation assay. Heparin (heparin/ 
siRNA weight ratios: 2, 10, 25 and 100) was mixed with BMs-PEI-siRNA and incubated for another $15 \mathrm{~min}$ at room temperature. The resultants were subjected to agarose gel electrophoresis (DYY-6C, Liuyi Biological Technology Co., Ltd, China).

To determine the serum stability assay, naked siRNA and BMs-PEI-siRNA nanocomposites were treated with $50 \%$ fetal bovine serum and incubated for $60,60,90,120$ and $150 \mathrm{~min}$. At predetermined time intervals, heparin was added to nanocomposites group, followed by incubation for $15 \mathrm{~min}$. All samples were loaded on $0.8 \%$ agarose gel electrophoresis for retardation analysis.

Further, the enzyme stability assay was performed by incubating naked siRNA and BMs-PEI-siRNA nanocomposites individually with RNase A for 60, 60, 90, 120 and $150 \mathrm{~min}$. The samples were then subjected to agarose gel electrophoresis.

\section{Cell viability assay}

The cytotoxicity of the designed nanoconjugates was measured using CCK-8 assay at a different siRNA concentration (dose-dependent) and incubation times (timedependent). HeLa cells were seeded into 96-well plates at $2 \times 10^{4}$ cells/well and incubated for proper cell attachment. After $24 \mathrm{~h}$ of incubation, the cells were subjected to treatment with siRNA, BMs-PEI, BMs-PEI-siRNA (STAT 3) and BMs-PEI-siRNA (NC) (the contents of siRNA were set as $2.5,5,7.5$ and $10 \mathrm{pmol}$ ) in $100 \mu \mathrm{L}$ of serum-free DMEM for $6 \mathrm{~h}$. The medium was then replaced with $200 \mu \mathrm{L}$ of DMEM containing 10\% FBS and incubated for further $48 \mathrm{~h}$. At the end of the incubation, $20 \mu \mathrm{L}$ of CCK- 8 reagent was added to each well and further incubated for $2 \mathrm{~h}$. Finally, the absorbance was recorded by using a microplate reader at $450 \mathrm{~nm}$ (Multiskan GO, Thermo Scientific Co., Ltd, USA).

Time-dependent assessment of cell viability was performed as described above by incubating the cells with samples [siRNA, BMs-PEI, BMs-PEI-siRNA (STAT 3) and BMs-PEI-siRNA (NC) (the content of siRNA was $5 \mathrm{pmol})$ ] at a different time periods 24,48 and $72 \mathrm{~h}$.

\section{Cell apoptosis assay}

To observe the cell apoptosis induced by BMs-PEIsiRNA nanocomposites, HeLa cells were seeded at a density of $1 \times 10^{5}$ cells/well in 24-well plates and incubated for $24 \mathrm{~h}$. Later, cells were treated with siRNA and BMs-PEI-siRNA (the concentration of siRNA was $50 \mathrm{nM}$ ) suspended in $0.5 \mathrm{~mL}$ serum-free DMEM for $6 \mathrm{~h}$ and then replaced with $1 \mathrm{~mL}$ DMEM containing $10 \%$ FBS and incubated for $48 \mathrm{~h}$. Subsequently, cells were harvested and washed three times with PBS, then $25 \mu \mathrm{L}$ of cell suspension was stained with $1 \mu \mathrm{L}$ of AO/EB dual stain reagent for 2-3 $\mathrm{min}$ in the dark according to the manufacturer's instructions. The apoptotic cells were analyzed by observing them under CLSM (Leica TCS SP8, Germany).

\section{Cellular uptake study}

HeLa cells were cultured on $35 \mathrm{~mm}$ glass-bottom dishes at a density of $4 \times 10^{5}$ cells/dish and incubated for $24 \mathrm{~h}$ for proper cell attachment. Cells were then treated with FAM-labeled BMs-PEI-siRNA nanocomposites (the concentration of siRNA was $50 \mathrm{nM}$ ) for 0.5 and $6 \mathrm{~h}$. After pirating the medium, the cells were washed thrice with cold PBS and then the cells were fixed with formaldehyde (4\%) for $10 \mathrm{~min}$, then washed and stained with DAPI. The dishes were eventually observed under CLSM (Leica TCS SP8, Germany).

\begin{abstract}
Abbreviations
AO/EB: acridine orange/ethidium bromide; N/P: nitrogen/phosphate; MDR: multidrug resistance; siRNA: small interfering RNA; RNase: ribonuclease; PEl: polyethyleneimine; APIs: active pharmaceutical agents; DLS: dynamic light scattering; TEM: transmission electron microscope; EPR: enhanced permeation and retention; RNAi: RNA interference; PBS: phosphate buffered saline; BMs: bacterial magnetosomes; BMs-PEI-siRNA: siRNA loaded BMs delivery system by using polyethyleneimine as a crosslinker; DEPC: diethyl pyrocarbonate; TAE: tris acetate EDTA; DMEM: Dulbecco's Modified Eagle's Medium; CLSM: confocal laser scanning microscope.
\end{abstract}

\section{Authors' contributions}

QD and YL conceived and designed the experiments. QD performed the experiment and compiled the manuscript. JW and WJ kindly presented the nanomaterials and gave helpful advice during the experiments and compilation of manuscripts. RL, RKK, and SW contributed to the data analysis and technical notes. All authors read and approved the final manuscript.

\section{Author details}

${ }^{1}$ College of Chemical Engineering, Huaqiao University, Xiamen 361021, People's Republic of China. ${ }^{2}$ Institute of Pharmaceutical Engineering, Huaqiao University, Xiamen 361021, People's Republic of China. ${ }^{3}$ Fujian Provincial Key Laboratory of Biochemical Technology, Huaqiao University, Xiamen 361021, People's Republic of China. ${ }^{4}$ State Key Laboratories for Agrobiotechnology and College of Biological Sciences, China Agricultural University, Beijing 100094, People's Republic of China.

\section{Acknowledgements}

We would like to thank the editor and reviewers for taking lots of time and giving constructive suggestions.

\section{Competing interests}

The authors declare that they have no competing interests.

\section{Availability of data and materials}

All data of this manuscript are present in the article.

\section{Consent for publication}

We transfer the rights and permissions of this work to the Microbial Cell Factories to publish this research article.

\section{Ethics approval and consent to participate} Not applicable.

\section{Funding}

Financial support from NSFC (31000441 and 31170939), National marine economic innovation and development project (16PYY007SF17), the Science Research Foundation of National Health and Family Planning Commission of 
PRC \& United Fujian Provincial Health and Education Project for Tacking the Key Research (WKJ2016-2-22), the Program for New Century Excellent Talents in Fujian Province University (2014FJ-NCET-ZR01) and the Promotion Program for Young and Middle-aged Teachers in Science and Technology Research of Huaqiao University (ZQN-PY108) are gratefully acknowledged.

\section{Publisher's Note}

Springer Nature remains neutral with regard to jurisdictional claims in published maps and institutional affiliations.

Received: 11 April 2017 Accepted: 20 November 2017

Published online: 28 November 2017

\section{References}

1. Kankala RK, Liu CG, Chen AZ, Wang SB, Xu PY, Mende LK, Liu CL, Lee CH, $\mathrm{Hu}$ YF. Overcoming multidrug resistance through the synergistic effects of hierarchical $\mathrm{pH}$-sensitive, ROS-generating nanoreactors. ACS Biomater Sci Eng. 2017;3(10):2431-42.

2. Yang WQ, Zhang Y. RNAi-mediated gene silencing in cancer therapy. Expert Opin Biol Ther. 2012;12(11):1495-504.

3. Manoharan M. RNA interference and chemically modified small interfering RNAs. Curr Opin Chem Biol. 2004:8(6):570-9.

4. Gandhi NS, Tekade RK, Chougule MB. Nanocarrier mediated delivery of siRNA/miRNA in combination with chemotherapeutic agents for cancer therapy: current progress and advances. J Control Release. 2014;194:238-56.

5. Xiao B, Ma LJ, Merlin D. Nanoparticle-mediated co-delivery of chemotherapeutic agent and siRNA for combination cancer therapy. Expert Opin Drug Deliv. 2017;14(1):65-73.

6. Egorova A, Shubina A, Sokolov D, Selkov S, Baranov V, Kiselev A. CXCR4targeted modular peptide carriers for efficient anti-VEGF siRNA delivery. Int J Pharm. 2016;515(1-2):431-40.

7. Weinstein S, Peer D. RNAi nanomedicines: challenges and opportunities within the immune system. Nanotechnology. 2010;21:232001.

8. Perevyazko IY, Bauer M, Pavlov GM, Hoeppener S, Schubert S, Fischer D, Schubert US. Polyelectrolyte complexes of DNA and linear PEl: formation, composition and properties. Langmuir. 2012;28(46):16167-76.

9. Kanthamneni N, Yung B, Lee RJ. Effect of eudragit on in vitro transfection efficiency of PEI-DNA complexes. Anticancer Res. 2016;36(1):81-5.

10. Xie LS, Tan Y, Wang ZY, Liu H, Zhang N, Zou C, Liu X, Liu G, Lu J, Zheng HR. Epsilon-caprolactone-modified polyethylenimine as efficient nanocarriers for siRNA delivery in vivo. ACS Appl Mater Interfaces. 2016;8(43):29261-9.

11. Ganas C, Weiss A, Nazarenus M, Rosler S, Kissel T, Gil PR, Parak WJ. Biodegradable capsules as non-viral vectors for in vitro delivery of PEl/siRNA polyplexes for efficient gene silencing. J Control Release. 2014;196:132-8.

12. Wang MX, Wu B, Tucker JD, Bollinger LE, Lu PJ, Lu QL. Poly(ester amine) composed of polyethylenimine and pluronic enhance delivery of antisense oligonucleotides in vitro and in dystrophic mdx mice. Mol Ther Nucleic acids. 2016;5(8):e341.

13. Park JW, Bae KH, Kim C, ParkTG. Clustered magnetite nanocrystals cross-linked with PEI for efficient siRNA delivery. Biomacromolecules. 2011;12:457-65.

14. Godbey WT, Mikos AG. Recent progress in gene delivery using non-viral transfer complexes. J Control Release. 2001;72:115-25.

15. Danafar H, Rostamizadeh K, Davaran S, Hamidi M. Drug-conjugated PLAPEG-PLA copolymers: a novel approach for controlled delivery of hydrophilic drugs by micelle formation. Pharm Dev Technol. 2017;22(8):947-57.

16. Apaolaza PS, Del Pozo-Rodríguez A, Solinís MA, Rodríguez JM, Friedrich U, Torrecilla J, Weber BH, Rodríguez-Gascón A. Structural recovery of the retina in a retinoschisin-deficient mouse after gene replacement therapy by solid lipid nanoparticles. Biomaterials. 2016;90:40-9.

17. Gharebaghi F, Dalali N, Ahmadi E, Danafar H. Preparation of wormlike polymeric nanoparticles coated with silica for delivery of methotrexate and evaluation of anticancer activity against MCF7 cells. J Biomater Appl. 2017;31:1305-16.
18. Kheiri MH, Sharafi A, Attari E, Danafar H. Pharmacokinetics and in vitro and in vivo delivery of sulforaphane by PCL-PEG-PCL copolymeric-based micelles. Artif Cells Nanomed Biotechnol. 2017;45(8):1728-39.

19. Manjili HK, Malvandi H, Mousavi MS, Attari E, Danafar $H$. In vitro and in vivo delivery of artemisinin loaded PCL-PEG-PCL micelles and its pharmacokinetic study. Artif Cells Nanomed Biotechnol. 2017;7:1-11.

20. Zhao Z, Li Y, Xie MB. Silk fibroin-based nanoparticles for drug delivery. Int J Mol Sci. 2015;16(3):4880-903.

21. Fan JQ, Liu YG, Wang SB, Liu YL, Li SM, Long RM, Zhang R, Kankala RK. Synthesis and characterization of innovative poly(lactide-co-glycolide)(poly-L-ornithine/fucoidan) core-shell nanocarriers by layer-by-layer self-assembly. RSC Adv. 2017;7:32786.

22. Sun JB, Li Y, Liang XJ, Wang PC. Bacterial magnetosome: a novel biogenetic magnetic targeted drug carrier with potential multifunctions. J Nanomater. 2011;2011:469031-43.

23. Bird SM, Galloway JM, Rawlings AE, Bramble JP, Staniland SS. Taking a hard line with biotemplating: cobalt-doped magnetite magnetic nanoparticle arrays. Nanoscale. 2015;7:7340-51.

24. Sun JB, Tang T, Duan JH, Xu PX, Wang ZL, Zhang YD, Wu LY, Li Y. Biocompatibility of bacterial magnetosomes: acute toxicity, immunotoxicity and cytotoxicity. Nanotoxicology. 2010;4(3):271-83.

25. Matsunaga T, Kamiya S. Use of magnetic particles isolated from magnetotactic bacteria for enzyme immobilization. Appl Microbiol Biotechnol. 1987;26(4):328-32.

26. Long RM, Liu YG, Dai QL, Wang SB, Deng QJ, Zhou X. A natural bacteriumproduced membrane-bound nanocarrier for drug combination therapy. Materials. 2016;9:889.

27. Li X, Wang B, Jin HL, Jiang W, Tian JS, Guan F, Li Y. Bacterial magnetic particles (BMPs)-PEl as a novel and efficient non-viral gene delivery system. J Gene Med. 2007;9(8):679-90.

28. Gorby YA, Beveridge TJ, Blakemore RP. Characterization of the bacterial magnetosome membrane. J Bacteriol. 1988;170:834-41.

29. Nakamura N, Matsunaga T. Highly sensitive detection of allergen using bacterial magnetic particles. Anal Chim Acta. 1993;281:585-9.

30. Kim B, Han G, Toley BJ, Kim CK, Rotello VM, Forbes NS. Tunning payload delievery in tumour cylindroids using gold nanoparticles. Nat Nanotechnol. 2010;5(6):465-72.

31. Ku SH, Jo SD, Lee YK, Kim K, Kim SH. Chemical and structural modifications of RNAi therapeutics. Adv Drug Deliv Rev. 2016;104:16-28.

32. Lin G, Zhu WC, Yang L, Wu J, Lin BB, Xu Y, Cheng ZZ, Xia CC, Gong QY, Song $B$, et al. Delivery of siRNA by MRI-visible nanovehicles to overcome drug resistance in MCF-7/ADR human breast cancer cells. Biomaterials. 2014;35:9495-507.

33. Shim MS, Kwon YJ. Acid-responsive linear polyethylenimine for efficient, specific, and biocompatible siRNA delivery. Bioconjug Chem. 2009;20(3):488-99.

34. Robb GB, Brown KM, Khurana J, Rana TM. Specific and potent RNAi in the nucleus of human cells. Nat Struct Mol Biol. 2005;12(2):133-7.

35. Sun JB, Duan JH, Dai SL, Ren J, Guo L, Jiang W, Li Y. Preparation and antitumor efficiency evaluation of doxorubicin-loaded bacterial magnetosomes: magnetic nanoparticles as drug carriers isolated from magnetospirillum gryphiswaldense. Biotechnol Bioeng. 2008;101(6):1313-20.

\section{Submit your next manuscript to BioMed Central and we will help you at every step:}

- We accept pre-submission inquiries

- Our selector tool helps you to find the most relevant journal

- We provide round the clock customer support

- Convenient online submission

- Thorough peer review

- Inclusion in PubMed and all major indexing services

- Maximum visibility for your research

Submit your manuscript at www.biomedcentral.com/submit 